\title{
Semi-Flocking-Controlled Mobile Sensor Networks for Tracking Targets with Different Priorities
}

\author{
Wanmai Yuan ${ }^{\dagger b}$, Nuwan Ganganath ${ }^{\natural}$, Chi-Tsun Cheng ${ }^{\S *}$, \\ Shahrokh Valaee ${ }^{\Uparrow}$, Guo Qing ${ }^{b}$, Francis C.M. Lau ${ }^{\dagger}$, and Herbert H.C. Iu ${ }^{\natural}$ \\ Email: yuanwanmai@hit.edu.cn nuwan@ganganath.lk ben.cheng@rmit.edu.au \\ valaee@ece.utoronto.ca qguo@hit.edu.com francis-cm.lau@polyu.edu.hk herbert.iu@uwa.edu.au \\ ${ }^{\dagger}$ Dept. of Electronic and Information Eng., The Hong Kong Polytechnic University, Hung Hom, Hong Kong

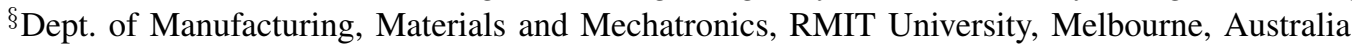 \\ ${ }^{\natural}$ School of Electrical, Electronic and Computer Eng., The University of Western Australia, Perth, Australia \\ ${ }^{b}$ School of Electronics and Information Eng., Harbin Institute of Technology, Harbin, P.R.China \\ ฯ Dept. of Electrical and Computer Eng., University of Toronto, Toronto, Canada
}

\begin{abstract}
Semi-flocking algorithms have been demonstrated to be efficient in maneuvering MSNs in multi-target tracking tasks. In many real-world applications, targets can be assigned with different priorities according to their importance of being tracked. However, existing semi-flocking algorithms normally assume the importance of all targets to be identical, which may not allocate resources in an efficient manner. In this paper, we propose a target evaluation method that incorporates priorities of the targets in the assessment process. Based on the evaluation results, mobile agents decide to track a target or continue to scan the terrain via a probabilistic task switching mechanism. Simulation results indicate a higher effectiveness of the proposed method in target tracking and area coverage when compared with two existing semi-flocking algorithms.
\end{abstract}

Index Terms-Semi-flocking, multi-target tracking, mobile sensor networks, target evaluation.

\section{INTRODUCTION}

Mobile sensor networks (MSNs) have been widely utilized for multiple targets tracking applications, such as unmanned border patrol, wild-animals monitoring, and search-rescue operations. Mobile agents can collect data of the surrounding environment via their sensors. Based on the collected information, they are capable of self-organizing and self-deploying themselves to deal with different tasks [1]. Furthermore, mobile agents can exchange information via local communications with other nearby agents. These agents can cooperate with their fellows to achieve desirable tracking performances [2]. To improve the efficiency of MSNs in multiple targets tracking applications, effective motion control mechanisms are needed for controlling agents to maximize their area coverage and enhance their target tracking capabilities.

In recent years, researches have been focusing on multiple targets tracking problems using MSNs. Dames et al. [3] considered to control teams of mobile agents to detect and locate multiple targets. In their work, their main innovation is by allowing agents to perform autonomous information fusion tasks. In [4] and [5], authors combined flocking control with an estimation strategy for multiple targets tracking. In [6], a clustering algorithm was proposed to organize agents into clusters, then a Munkres algorithm is applied to address the target tracking and data association problems. In order to reduce energy expenditure in target tracking, energy-efficient target tracking algorithms have been investigated in [7] and [8]. Energy-efficiency in collaborative target tracking has been further surveyed and summarized in [9].

Semi-flocking algorithms [10]-[12] have been demonstrated to be efficient in controlling mobile agents in tracking multiple targets. The concept of semi-flocking is to form a small number of agents around each target while keeping the remaining agents to scan the area of interest (AoI) for newly emerging targets. For agents that are tracking targets, they are controlled by a flocking algorithm [13]. At the meanwhile, agents which are sensing the AoI are navigated by an anti-flocking algorithm [14]. Semi-flocking-controlled agents are capable of simultaneously providing wide area sensing coverage and tracking multiple targets. In many real-world target tracking scenarios, targets can have different priorities due to their attributes and specific requirements. Such a criterion, however, is omitted in existing semi-flocking algorithms.

The focus of this paper is on developing a semi-flocking algorithm to track multiple targets with different priorities in an AoI. A new target evaluation method is proposed for agents to assess each target. Based on the evaluation results, agents can track a target or keep sensing the AoI via a probabilistic task switching mechanism. The proposed semiflocking algorithm provides better practicability for applying MSNs in real-world target tracking applications.

The rest of the paper is organized as follows. Section II presents some preliminaries of MSNs and semi-flocking control. In Section III, the proposed semi-flocking algorithm is introduced and elaborated. Simulation results are presented and discussed in Section IV. Finally, concluding remarks are provided in Section V.

\section{PRELIMINARIES}

\section{A. Motion Control of Mobile Sensor Networks}

Consider an MSN consisting of $N$ agents in a rectangular AoI with width $w$ and length $l$. The motion of agent $i$ is 
controlled by

$$
\left\{\begin{array}{l}
\dot{q}_{i}=p_{i}, \\
\dot{p}_{i}=u_{i}, \quad i=1,2, \ldots, N,
\end{array}\right.
$$

where $q_{i}$ and $p_{i}$ are the position and velocity of agent $i$, respectively, while $u_{i}$ is the control input of agent $i$.

Each agent has a limited communication capability that it can only exchange information with other agents within its communication radius $r_{\mathrm{c}}$ [10], [14]. The set of neighbors of agent $i$ is represented as

$$
\mathcal{N}_{i}=\left\{j:\left\|q_{j}-q_{i}\right\|<r_{\mathrm{c}}, j=1,2, \ldots, N, j \neq i\right\},
$$

where $q_{j}$ is the position of agent $j$ and $\|\cdot\|$ is the Euclidean norm [10].

\section{B. Semi-Flocking Control}

Semi-flocking-controlled agents are capable of sensing the AoI and tracking targets simultaneously. Initially, all agents focus on maximizing their cumulative area coverage and operating in their sensing mode. These agents are controlled by three anti-flocking rules, namely decentering, collision avoidance, and selfishness. The control input of agent $i$ in sensing mode consists of three terms, such that

$$
u_{i}=f_{i}^{g}+f_{i}^{d}+f_{i}^{s} .
$$

The first term $f_{i}^{g}[11]$ is used to regulate the distance between agents and is given as

$$
f_{i}^{g}=\sum_{j \in \mathcal{N}_{i}} \phi_{\alpha}\left(\left\|q_{j}-q_{i}\right\|_{\sigma}\right) n_{i j}
$$

where $n_{i j}$ is a vector along the line connecting $q_{i}$ and $q_{j}$ which is given as $n_{i j}=\left(q_{j}-q_{i}\right) / \sqrt{1+\epsilon\left\|q_{j}-q_{i}\right\|^{2}}$ with $\epsilon \in(0,1)$. Here, the action function $\phi_{\alpha}(x)$ [15] is denoted as

$$
\phi_{\alpha}(x)=\phi\left(x-d_{\alpha}\right) \rho_{h}\left(\frac{x}{r_{\alpha}}, h\right),
$$

where

$$
\phi(y)=\frac{1}{2}\left[\left(\lambda_{1}+\lambda_{2}\right) \Gamma\left(y+\lambda_{3}\right)+\left(\lambda_{1}-\lambda_{2}\right)\right] .
$$

In (5), $d_{\alpha}$ is a positive constant and $r_{\alpha}=\left\|r_{\mathrm{c}}\right\|_{\sigma}$, where $\sigma$ norm of $\omega$ is given as $\|\omega\|_{\sigma}=\left[\sqrt{1+\epsilon\|\omega\|^{2}}-1\right] / \epsilon$. In (6), $\Gamma(\varrho)=\varrho / \sqrt{1+\varrho^{2}}$. The parameters $\lambda_{1}, \lambda_{2}$, and $\lambda_{3}$ satisfy $0<\lambda_{1} \leq \lambda_{2}$ and $\lambda_{3}=\left|\lambda_{1}-\lambda_{2}\right| / \sqrt{4 \lambda_{1} \lambda_{2}}$ [11]. The bump function $\rho_{h}(\xi, h)$ [11] is defined as

$$
\rho_{h}(\xi, h)= \begin{cases}1, & \text { if } \xi \in[0, h), \\ \frac{1}{2}\left[1+\cos \left(\frac{\pi(\xi-h)}{1-h}\right)\right], & \text { if } \xi \in[h, 1], \\ 0, & \text { otherwise }\end{cases}
$$

with $h \in(0,1)$.

The second term $f_{i}^{d}$ is a velocity consensus term [11] which is given as

$$
f_{i}^{d}=\sum_{j \in \mathcal{N}_{i}}\left(p_{j}-p_{i}\right) a_{i j}\left(q_{i}, q_{j}\right),
$$

where $p_{j}$ is the velocity of agent $j$. The spatial adjacency matrix $a_{i j}\left(q_{i}, q_{j}\right)[11]$ is expressed as

$$
a_{i j}\left(q_{i}, q_{j}\right)=\rho_{h}\left(\frac{\left\|q_{j}-q_{i}\right\|_{\sigma}}{r_{\alpha}}\right) \in[0,1], \quad i \neq j .
$$

Furthermore, the third term $f_{i}^{s}$ is a selfishness term [15] that navigates agents to areas that have not been covered recently and is defined as

$$
f_{i}^{s}=b_{1} \frac{\left(q_{i}^{\eta}-q_{i}\right)}{\left\|q_{i}^{\eta}-q_{i}\right\|}-b_{2} p_{i}
$$

where $b_{1}$ and $b_{2}$ are positive constants. Here, $q_{i}^{\eta}$ is the next sensing candidate of agent $i$ which is selected based on the following steps. The AoI is divided into a number of square patches. Each agent records the information about "when a patch was last visited" into its information map. An agent can update its information map by actually providing sensing coverage to a patch or with information maps from neighboring agents via single-hop communications. Then, among the patches with the longest time not being visited, an agent chooses the one with the shortest total distance to both its current position and its current sensing goal as the next sensing candidate $q_{i}^{\eta}$ [14].

When an agent $i$ detects a target $k$ and it decides to track this target, it will switch into tracking mode. For agents in tracking mode, they will be controlled by three flocking rules, namely collision avoidance, velocity matching, and flock centering. Its control input $u_{i}$ is expressed as

$$
u_{i}=f_{i}^{g}+f_{i}^{d}+f_{i}^{t}
$$

where the target tracking term $f_{i}^{t}$ is given as

$$
f_{i}^{t}=b_{3}\left(q_{k}-q_{i}\right)+b_{4}\left(p_{k}-p_{i}\right)
$$

where $b_{3}$ and $b_{4}$ are positive constants. Furthermore, $q_{k}$ and $p_{k}$ are the position and velocity of target $k$ with $1 \leq k \leq M$, respectively. Here, $M$ is the number of targets in the AoI.

\section{Proposed SEMI-Flocking Algorithm}

In real-world target tracking tasks, such as hazards detection and animals surveillance, targets have different priorities due to their attributes and requirements. For targets with higher priorities, more agents should be allocated to track them promptly. In contrast, targets with lower priorities should then be handled with relatively fewer agents. To achieve that, a semi-flocking algorithm for tracking multiple targets with different priorities is proposed in this section.

\section{A. Target Evaluation Method}

Initially, all agents begin with operating in their sensing mode and searching for new targets in the AoI. When a target is detected by an agent via its sensor or local communications with nearby agents, it will evaluate the target based on five factors: 1) the minimum number of required agents for tracking the target $n_{\min }(k) ; 2$ ) the maximum number of required agents for tracking the target $\left.n_{\max }(k) ; 3\right)$ the time duration since the target was last tracked by at least $n_{\min }(k)$ agents 
$\left.T_{\mathrm{n}}(k) ; 4\right)$ the number of agents which are currently tracking the target $n_{i}(k)$; and 5) the distance between the target and the current agent. The evaluation function of agent $i$ on target $k$ is defined as

$$
\begin{aligned}
e(i, k)= & \left(\frac{T_{\mathrm{n}}(k)}{\mu T_{\mathrm{n}}(k)+T_{\mathrm{s}}}+1-\frac{1}{\mu}\right) \times \rho_{h}\left(\frac{n_{i}(k)}{n_{\max }(k)}, \frac{n_{\min }(k)}{n_{\max }(k)}\right) \\
& \times \rho_{h}\left(\frac{\left\|q_{k}-q_{i}\right\|}{r_{\max }}, \frac{r_{\mathrm{s}}}{r_{\max }}\right),
\end{aligned}
$$

where $e(i, k)$ is the evaluation result of agent $i$ on target $k$. Note that $n_{i}(k)$ is obtained by agent $i$ via local communications with its nearby agents. The sensing range $r_{\mathrm{s}}$ is identical for all agents while $r_{\max }>r_{\mathrm{s}}$. Furthermore, $\mu$ and $T_{\mathrm{s}}$ are two positive system tuning parameters.

In (10), the first term $\frac{T_{\mathrm{n}}(k)}{\mu T_{\mathrm{n}}(k)+T_{\mathrm{s}}}+1-\frac{1}{\mu}$ is used to give higher preferences to targets that have not being tracked by enough agents for a long duration of time. If target $k$ is tracked by at least $n_{\min }(k)$ agents at the moment, then $T_{\mathrm{n}}(k)=0$. Otherwise, $T_{\mathrm{n}}(k)=T-T_{\mathrm{e}}(k)$, where $\mathrm{T}$ is the current system time and $T_{\mathrm{e}}(k)$ is the time when target $k$ emerges in the AoI.

The second term $\rho_{h}\left(\frac{n_{i}(k)}{n_{\max }(k)}, \frac{n_{\min }(k)}{n_{\max }(k)}\right)$ prioritizes the targets with a few number of their tracking agents. When the number of agents tracking target $k$ is lower than $n_{\min }(k)$, the value of the second term is equal to 1 , which indicates a strong demand for tracking agents from target $k$. When $n_{\min }(k) \leq n_{i}(k) \leq$ $n_{\max }(k)$, the need for acquiring additional tracking agents will decline continuously. Finally, when $n_{i}(k)>n_{\max }(k)$, there is no need of more agents to tract target $k$.

The third term $\rho_{h}\left(\frac{\left\|q_{k}-q_{i}\right\|}{r_{\max }}, \frac{r_{\mathrm{s}}}{r_{\max }}\right)$ is used to prioritize the targets according to their distances from agent $i$. When the distance between target $k$ and agent $i$ is shorter than the sensing range $r_{\mathrm{s}}$, the value of the third term is equal to 1 . The value will decrease gradually with the raise of $q_{k}-q_{i}$. If $q_{k}-q_{i}$ is larger than $r_{\max }$, agent $i$ will not navigate toward target $k$.

Note that the outcomes of (10) vary between 0 to 1 . The design rationale of such a target evaluation method is to regulate the size of the tracking cluster for each target. Furthermore, it can enable more agents to track targets with high priorities.

\section{B. Decision-Making Mechanism}

After agent $i$ obtained the evaluation result of target $k$, this result will be used to calculate the transition probability for agent $i$ switching between tracking a target or keeping sensing the AoI [15], such that

$$
P_{i k}= \begin{cases}e(i, k), & \text { if } 0 \leq \sum_{m=1}^{M} e(i, m) \leq 1, \\ \frac{e(i, k)}{\sum_{m=1}^{M} e(i, m)}, & \text { if } \sum_{m=1}^{M} e(i, m)>1,\end{cases}
$$

and

$$
P_{i 0}= \begin{cases}1-\sum_{m=1}^{M} e(i, m), & \text { if } 0 \leq \sum_{m=1}^{M} e(i, m) \leq 1, \\ 0, & \text { if } \sum_{m=1}^{M} e(i, m)>1 .\end{cases}
$$

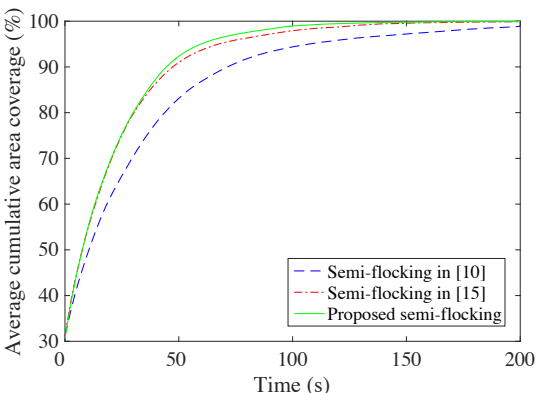

Fig. 1. Average cumulative coverage of MSNs with 30 agents in the AoI. All the data points presented are the results of averaging over 50 simulations.

TABLE I

PARAMETER SETTINGS

\begin{tabular}{c|c|c|c|c|c|c|c|c|c|c|c|c|c|c} 
Parameters & $n_{\min }(k)$ & $r_{\mathrm{s}}$ & $r_{\mathrm{c}}$ & $\epsilon$ & $\lambda_{1}$ & $\lambda_{2}$ & $h$ & $b_{1}$ & $b_{2}$ & $b_{3}$ & $b_{4}$ & $\mu$ & $T_{\mathrm{s}}$ & $r_{\max }$ \\
\hline Values & 3 & $10 \mathrm{~m}$ & $18 \mathrm{~m}$ & 0.1 & 5 & 5 & 0.2 & 0.1 & 0.6 & 0.05 & 0.05 & 2 & 100 & 75
\end{tabular}

When $0 \leq \sum_{m=1}^{M} e(i, m) \leq 1$, the evaluation result will directly become the transition probability for agent $i$ in the sensing mode to switch into tracking target $k$. At the meanwhile, there is a probability with $P_{i 0}=1-\sum_{m=1}^{M} e(i, m)$ that agent $i$ remains in sensing mode. Alternatively, if $\sum_{m=1}^{M} e(i, m)>1$, then $P_{i k}=\frac{e(i, k)}{\sum_{m=1}^{M} e(i, m)}$ and $P_{i 0}=0$. Based on the transition probabilities, agent $i$ can make a Markov decision to select one of the targets to track or continue to sense the AoI. Furthermore, agents in tracking mode will return to sensing mode when their targets disappear from the AoI.

\section{Simulations}

\section{A. Simulation Setup}

The performances of the proposed semi-flocking algorithm were evaluated against the semi-flocking algorithms proposed in [10] and [15]. For all algorithms under test, including the ones in [10] and [15], their parameters in TABLE I remain constant. There are 2 randomly moving targets within the AoI. The maximum required numbers of agents for tracking the 2 targets are 4 and 8 , respectively. Initial positions of agents and targets were selected uniformly at random within the $150 \times$ $150 \mathrm{~m}^{2}$ AoI, while initial velocities of agents and targets were selected uniformly at random from $[-10,10]^{2} \mathrm{~ms}^{-1}$.

\section{B. Simulation Results}

In the first set of simulations, there are 30 agents in the AoI. Fig. 1 illustrates that MSNs controlled by the proposed semiflocking algorithm can complete searching the AoI faster than those in [10] and [15]. Agents controlled by the algorithm in [10] are easily trapped in local regions since they only use the visiting information on their 8 neighboring patches. With the help of information exchanges on the last visiting time of each patch, agents controlled by the algorithm in [15] are capable of visiting the patches which have not been visited for long durations. However, these agents ignore the priorities on the targets, such that each target was treated equally and tracked 


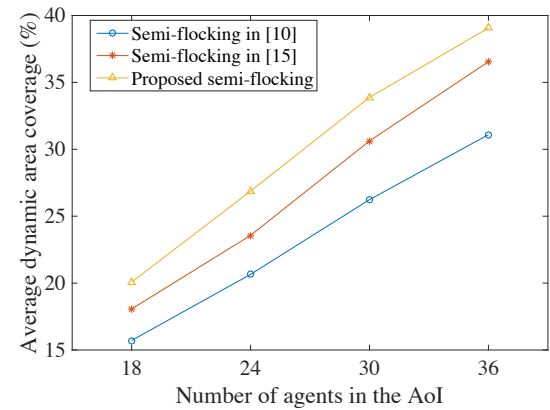

Fig. 2. Average dynamic area coverage of MSNs in 200 seconds with different number of nodes. All the data points presented are the results of averaging over 50 simulations.

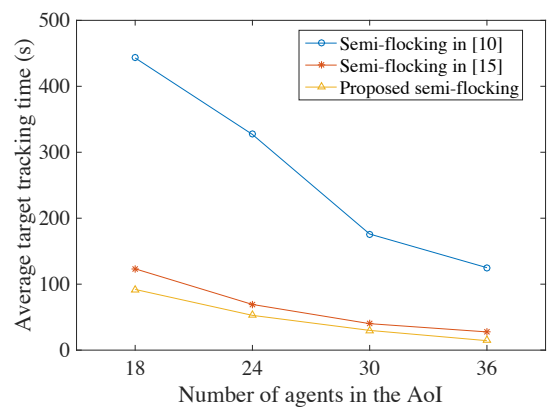

Fig. 3. Average target tracking time of the MSN with different number of nodes. All the data points presented are the results of averaging over 50 simulations.

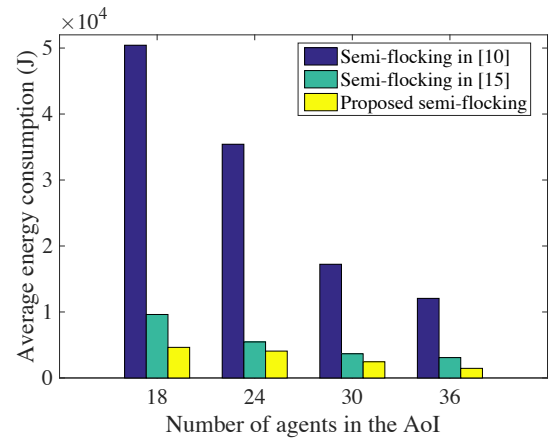

Fig. 4. Average energy consumption of the MSN for tracking all targets in the AoI with different number of nodes. All the data points presented are the results of averaging over 50 simulations.

by the same number of agents. In order to guarantee that all targets can be tracked by sufficient numbers of agents, more than enough agents were tracking the targets with the lower priority, which jeopardizes the area coverage capabilities of the MSNs. In contrast, MSN with the proposed algorithm not only can efficiently visit areas where have not been visited for a long time, it can allocate adequate numbers of agents for tracking multiple targets with different priorities. Thus, it can achieve a full scan of the AoI in a shorter time.

The next set of simulations was carried out to compare the dynamic area coverage performances of MSNs with different semi-flocking algorithms. The number of agents in the AoI was varied from 18 to 36 . According to the results in Fig. 2, it is obvious that the dynamic area coverages of MSNs increase with the number of agents. Since there are only two targets moving in the AoI, the numbers of agents for tracking these targets are fixed. By adding extra agents to the system, more agents will then be operating in their sensing mode. Therefore, an increased number of agents will yield a higher dynamic area coverage. Furthermore, it is observed that MSN with the proposed algorithm can achieve a relatively higher dynamic area coverage when compared to those in [10] and [15]. As explained above, agents controlled by the proposed algorithm are capable of maximizing their area coverage while minimize overlaps with others due to their information records and local communications with other agents.

The third set of simulations was conducted to analyze the target tracking capabilities of the proposed algorithm. The number of agents in the AoI was again varied from 18 to 36. Figs. 3 and 4 illustrate that MSN controlled by the proposed algorithm required shorter time and lower energy to track all the targets in the AoI. Note in this work, only the energy consumption due to motion of agents was taken into account. In the semi-flocking algorithm in [10], area coverage capabilities of agents have been limited by their searching strategies, in which agents only utilize the visiting information of their 8 neighboring patches. Furthermore, their agents do not exchange information on both areas and targets, which further reduce their target detecting efficiency. In the semiflocking algorithm in [15], although information maps and information lists were exchanged among agents, all targets were treated equally and more than enough agents were allocated to track targets with low priorities. In contrast, under the control of the proposed algorithm, agents evaluate targets in terms of their priorities and different number of agents were assigned to track each target. In MSNs with the proposed algorithm, agents can navigate toward their targets swiftly with lower energy consumption.

\section{CONClusion}

In this paper, a semi-flocking algorithm is proposed for MSNs to allocate sensing resource to multiple targets with different priorities. Agents in an MSN use a probabilistic task switching mechanism to switch between sensing and tracking modes. Under the control of the proposed algorithm, more agents will be allocated to targets with high priorities, and vice versa. Simulation results verify that the proposed algorithm is more capable in providing the required sensing coverage and target tracking capabilities than two existing semi-flocking algorithms.

\section{ACKNOWLEDGMENT}

This work is supported by the Department of Electronic and Information Engineering, the Hong Kong Polytechnic University (Project RUWM). 


\section{REFERENCES}

[1] N. Ganganath, W. Yuan, C.-T. Cheng, T. Fernando, and H. H. C. Iu, "Territorial marking for improved area coverage in anti-flocking-controlled mobile sensor networks," in 2018 IEEE International Symposium on Circuits and Systems (ISCAS). IEEE, 2018, pp. 1-4.

[2] H. Rezaee and F. Abdollahi, "A decentralized cooperative control scheme with obstacle avoidance for a team of mobile robots," IEEE Transactions on Industrial Electronics, vol. 61, no. 1, pp. 347-354, 2014.

[3] P. M. Dames and V. Kumar, "Autonomous localization of an unknown number of targets without data association using teams of mobile sensors." IEEE Transactions on Automation Science and Engineering, vol. 12, no. 3, pp. 850-864, 2015.

[4] Z. Wang and D. Gu, "Cooperative target tracking control of multiple robots," IEEE Transactions on Industrial Electronics, vol. 59, no. 8, pp. 3232-3240, 2012.

[5] H. Su, Z. Li, and M. Z. Chen, "Distributed estimation and control for two-target tracking mobile sensor networks," Journal of the Franklin Institute, vol. 354, no. 7, pp. 2994-3007, 2017.

[6] P. Parmar and M. Zaveri, "Multiple target tracking and data association in wireless sensor network," in 2012 Fourth International Conference on Computational Intelligence and Communication Networks (CICN). IEEE, 2012, pp. 158-163.

[7] Z. Liao, J. Wang, S. Zhang, J. Cao, and G. Min, "Minimizing movement for target coverage and network connectivity in mobile sensor networks," IEEE Transactions on Parallel and Distributed Systems, vol. 26, pp. 1971-1983, 2014.

[8] Y. Qi, P. Cheng, J. Bai, J. Chen, A. Guenard, Y.-Q. Song, and Z. Shi, "Energy-efficient target tracking by mobile sensors with limited sensing range." IEEE Transactions on Industrial Electronics, vol. 63, no. 11, pp. 6949-6961, 2016.

[9] O. Demigha, W.-K. Hidouci, and T. Ahmed, "On energy efficiency in collaborative target tracking in wireless sensor network: A review," IEEE Communications Surveys \& Tutorials, vol. 15, no. 3, pp. 1210-1222, 2013.

[10] S. H. Semnani and O. A. Basir, "Semi-flocking algorithm for motion control of mobile sensors in large-scale surveillance systems," IEEE Transactions on Cybernetics, vol. 45, no. 1, pp. 129-137, 2015.

[11] W. Yuan, N. Ganganath, C.-T. Cheng, S. Valaee, Q. Guo, and F. C. M Lau, "Energy-efficient semi-flocking control of mobile sensor networks on rough terrains," IEEE Transactions on Circuits and Systems II: Express Briefs, 2018, Early Access.

[12] W. Yuan, N. Ganganath, C.-T. Cheng, G. Qing, and F. C. M. Lau, "Path planning for semi-flocking-controlled mobile sensor networks on mobility maps," in 2018 IEEE International Symposium on Circuits and Systems (ISCAS). IEEE, 2018, pp. 1-5.

[13] R. Olfati-Saber, "Flocking for multi-agent dynamic systems: Algorithms and theory," IEEE Transactions on Automatic Control, vol. 51, no. 3 , pp. 401-420, 2006.

[14] N. Ganganath, C.-T. Cheng, and C. K. Tse, "Distributed antiflocking algorithms for dynamic coverage of mobile sensor networks," IEEE Transactions on Industrial Informatics, vol. 12, no. 5, pp. 1795-1805, 2016.

[15] W. Yuan, N. Ganganath, C.-T. Cheng, G. Qing, and F. C. M. Lau, "Semiflocking-controlled mobile sensor networks for dynamic area coverage and multiple target tracking," IEEE Sensors Journal, vol. 18, no. 21, pp. 8883-8892, 2018 